\title{
OBSERVACIONES PARA EL ESTUDIO DE LA RELACIÓN GENEALÓGICA ENTRE LA LENGUA PÁEZ Y LAS FAMILIAS CHOCÓ Y BARBACOA
}

\author{
Carla Victoria Jara Murillo
}

\begin{abstract}
RESUMEN
El propósito de este trabajo es buscar posibles cognados entre lenguas barbacoas y la lengua páez, ya que se ha propuesto que el páez pertenece a la familia barbacoa. Además, se buscarán posibles cognados entre el protochocó y el páez, con el fin de establecer si se vislumbra una relación entre esta lengua y la familia chocó.

Palabras clave: lenguas indígenas, lingüística histórica, lengua páez, familia chocó, familia barbacoa.
\end{abstract}

\section{ABSTRACT}

The purpose of this paper is to look for possible cognates between Barbacoan languages and the Paez language, since it has been proposed that Paez belongs to the Barbacoan family. Furthermore, possible cognates between Protochocoan and Paez will also be observed, in order to determine whether a relationship between Paez and the Chocoan family can be pursued.

Key words: indigenous languages, historical linguistics, Paez language, Barbacoan family, Chocoan family.

\section{Objetivo}

El objetivo de este trabajo es comparar datos de la lengua páez de Colombia con datos de lenguas de la familia barbacoa y de la familia chocó. Probablemente debido a su cercanía geográfica, se ha propuesto que el páez pertenece a la familia barbacoa sin que se haya presentado evidencia lingüística de ello. Por otra parte, se buscará establecer posibles cognados entre el páez y formas de las lenguas chocoes, en particular de la lengua huaunana y formas reconstruidas del protochocó.

Dra. Carla Victoria Jara M. Profesora, Escuela de Filología, Lingüística y Literatura. Directora Instituto de Investigaciones Lingüísticas. Universidad de Costa Rica.San Pedro de Montes de Oca, San José, Costa Rica 


\section{Fuentes}

Las fuentes que sirven de base para este trabajo son:

a) Vocabulario Comparativo: Palabras Selectas de Lenguas Indígenas de Colombia / Comparative Vocabulary: Selected Words in Indigenous Languages of Colombia (Huber \& Reed 1992). Aquí se recopilan términos en 68 lenguas indígenas de Colombia según las listas de palabras diseñadas por Morris Swadesh y John Rowe. Los datos han sido recogidos en su mayoría por lingüistas que trabajan con el Instituto Lingüístico de Verano en Colombia ${ }^{1}$. En lo sucesivo, esta fuente será identificada mediante la sigla VC.

b) Las Lenguas del Área Intermedia: Introducción a su Estudio Areal (Constenla Umaña 1991). En esta obra, Constenla estudia las relaciones entre las familias de lenguas del Área Intermedia con base en los métodos lexicoestadístico y aglomerativo ${ }^{2}$. En el presente estudio esta fuente será identificada mediante la sigla LAI.

c) Elementos de fonología comparada chocó (Constenla y Margery 1991). En este trabajo comparativo, en lo sucesivo identificado como EFCC, Constenla y Margery aportan la reconstrucción del sistema fonológico del protochocó y la reconstrucción de 311 formas entre protochocoes y protoemberaes, datos que se utilizan en el presente estudio para buscar coincidencias entre el páez y la familia chocó.

\section{Familias y lenguas del estudio}

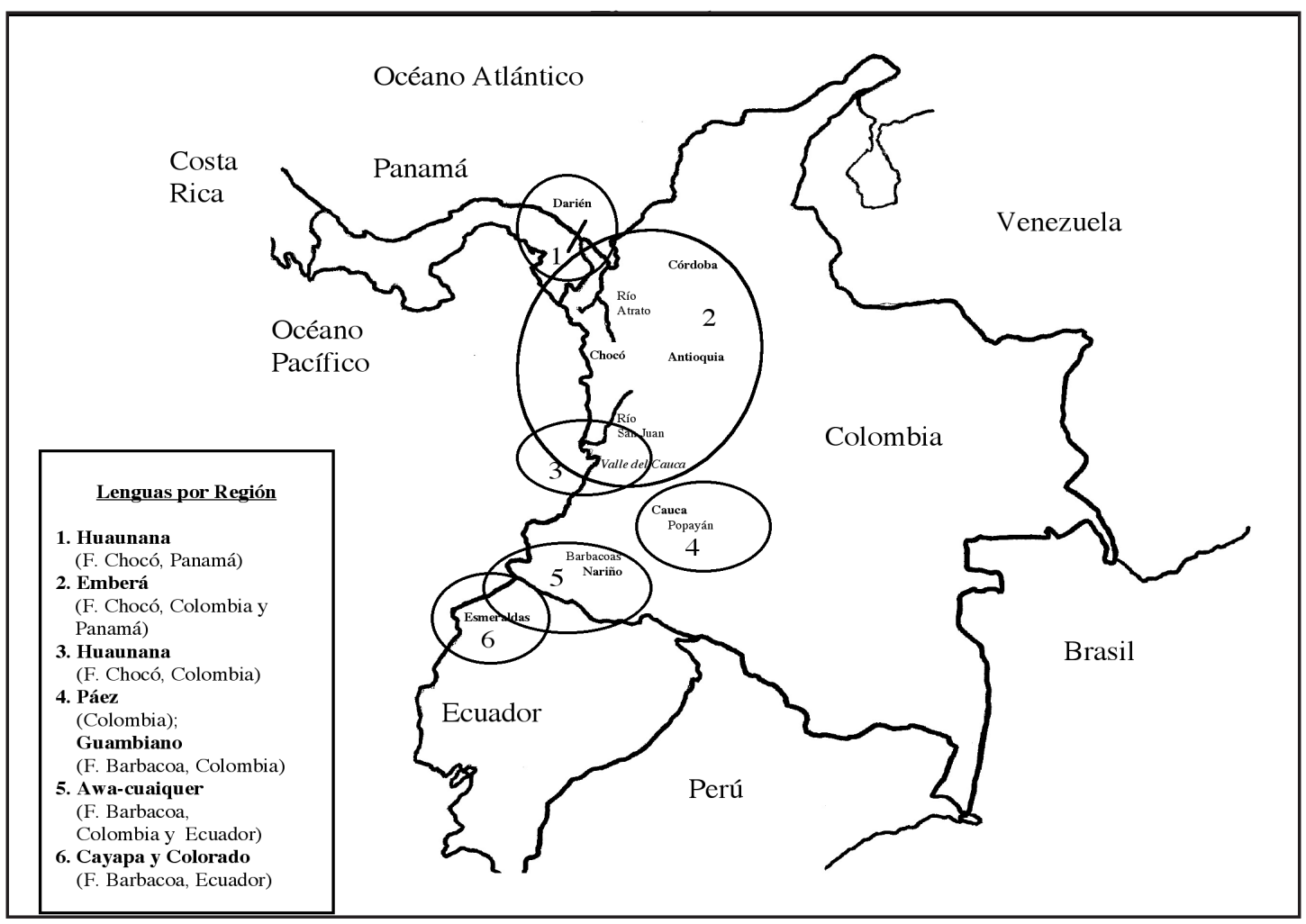


Fundamentalmente, las lenguas que se van a considerar en este trabajo son las siguientes:

a) las lenguas awa-cuaiquer y guambiano de la familia barbacoa;

b) la lengua páez;

c) la lengua huaunana y el protochocó de la familia chocó.

\subsection{La familia barbacoa}

A partir del grupo chibcha que desde 1888 había establecido Max Uhle (1890) y del grupo páez, propuesto por Paul Rivet y colaboradores en 1924, Loukotka (1968) incluyó en un solo tronco o cepa de lenguas (stock) denominado Chibcha, entre otros 18, los subgrupos páez, coconuco (con las lenguas guambiano y totoró) y barbacoa (con el awa-cuaiquer). Esta agrupación, actualmente considerada errónea, como se verá más adelante, ha influido sin embargo en varias clasificaciones posteriores. Tal como Landaburu (2000) señala con respecto a la clasificación de Loukotka, "(e)l primer gran conjunto de lenguas cuya inclusión en la agrupación chibcha cabe poner en duda es el de las lenguas del sur andino: páez, grupo coconuco, grupo barbacoa (...). Este encasillamiento fue obra de Paul Rivet, en un artículo famoso de 1910 (Beuchat \& Rivet 1910) cuya metodología no es aceptable según los criterios de hoy".

Una clasificación que hace eco a la de Loukotka en este aspecto es la de Greenberg (1987), quien propone un macrofilo compuesto de dos mesofilos, chibcha y paezano. La lenguas respectivas se distribuyen por América Central y la región norte de América del Sur. En el filo "paezano nuclear" (paezan nuclear) según su propia denominación, Greenberg incluye tres familias de lenguas (VC: xi):

1. chocó

2. barbacoa (lenguas awa (cuaiquer), cha'palaachi (cayapa) y tsafiqui pila (colorado))

3. páez (lenguas guambiano, páez y totoró)

Estos tres grupos quedan excluidos del ámbito chibcha gracias al trabajo lexicoestadístico de Constenla Umaña (1985). Landaburu, acogiendo la tesis de Constenla Umaña, señala que "mientras no se presenten argumentos válidos, deben considerarse como estirpes distintas [del chibcha]:

- El páez con sus dialectos (entre los cuales está el paniquitá)

- El grupo coconuco con el guambiano y el totoró que no son muy diferenciados y que probablemente convendría reducir a variantes dialectales

- El awa o cuaiquer, cuya cercanía al cayapa y colorado ecuatorianos queda por demostrar, para poder asentar el tradicional grupo barbacoa de la literatura" (Ibíd.).

También la disociación del paezano nuclear de Greenberg se ha ido imponiendo. Para el VC, “agrupar las lenguas CHOCÓ con PÁEZ es muy controvertido. La lista del ICC [Instituto Caro y Cuervo] incluye sólo el awa en la familia [barbacoa, con respecto a Colombia]; las lenguas colombianas incluidas en el grupo PAEZAN de Greenberg aparecen en la lista del ICC como no clasificadas [vg. guambiano y páez]" (VC: xiv). De acuerdo con Constenla Umaña (1991), es igualmente controversial agrupar las lenguas barbacoas con el páez. Es objetivo de este estudio precisamente indagar estas relaciones con base en la comparación de términos de las lenguas en cuestión. 
La clasificación que presenta el VC comienza con la de Grimes (1988), según la cual la familia barbacoa comprende las siguientes lenguas (entre paréntesis la clasificación de Grimes 1988):

1. awa (macro-chibcha, chibcha, barbacoa, pasto)

2. cha' palaachi ${ }^{3}$ (macro-chibcha, chibcha, barbacoa, cayapa-colorado)

3. tsafiqui pila ${ }^{4}$ (macro-chibcha, chibcha, barbacoa, cayapa-colorado) lia páez:

De seguido, el VC (loc. cit.) se refiere a las lenguas que Greenberg incluye en la fami-

4. guambiano (macro-chibcha, páez, inter-andino, coconuco)

5. páez (macro-chibcha, páez, inter-andino, panaquita)

6. totoró (no incluido en Grimes 1988).

Para la edición no. 14 de Ethnologue (Grimes 2000), la familia barbacoa aparece compuesta de la siguiente manera (el páez se enlista como un grupo aparte de una sola lengua):

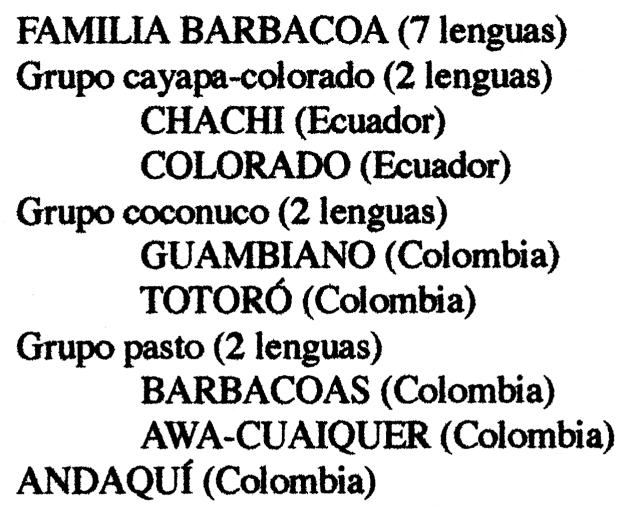

(http://www.ethnologue.com/show_family.asp?subid=2039)

De acuerdo con LAI, las lenguas de la familia barbacoa son cuatro y se sitúan en el área que se extiende del noroeste del Departamento del Cauca, Colombia, hasta la Provincia de Pichincha, Ecuador: guambiano, (awa)-cuaiquer, cayapa y colorado. LAI afirma que es incorrecta la relación que se ha propuesto entre el guambiano y el páez, es decir, el supuesto grupo coconuco, y en particular la tesis de una relación más estrecha entre ellas que entre el guambiano y las otras dos lenguas barbacoas: cayapa y colorado (por ejemplo en Loukotka 1968, Greenberg 1987, Swadesh 1959).

La población barbacoa, según las fuentes de LAI, es la siguiente:

Guambianos: 10.180, Colombia.

Cuaiqueres: 4.366 en Colombia, 951 en Ecuador.

Cayapas: 3.000 , Ecuador.

Colorados: 1200, Ecuador.

Referencias a los materiales descriptivos de las lenguas barbacoas se encuentran en Loukotka (1968) y Tovar (1961), que consignan materiales de hasta 1960; posteriores a esta fecha, en Wares (1985).

Con respecto a la diacronía de las lenguas barbacoas, LAI señala que Brinton (1891) establece la correcta integración de la familia. Beuchat y Rivet (1910) la modifican disocian- 
do el guambiano y relacionándolo con el páez equivocadamente. Como ya se vio, Loukotka (1968), Greenberg (1987) y Grimes (1988) prosiguieron con el error en sus clasificaciones. En LAI, Constenla utiliza listas comparativas de 14 rubros de las lenguas colorado, cuaiquer, cayapa, guambiano y páez, y agrega comentarios pertinentes con miras a esclarecer el error. Además, establece la clasificación interna de la familia barbacoa con base en el análisis lexicoestadístico y produce árboles de relaciones utilizando el análisis aglomerativo (programa SPSS, métodos bavrage, single linkage y complete linkage) del siguiente modo (LAI: 75):

\section{Familia barbacoa:}

Rama barbacoa septentrional: cuaiquer, guambiano.

Rama barbacoa meridional: colorado, cayapa.

Separaciones temporales:

Colorado y cayapa: 1.263 años

Cuaiquer y guambiano:3.349 años

Colorado y cuaiquer: 3.413 años

Colorado y guambiano:3.769 años

Cayapa y cuaiquer 3.769 años

Cayapa y guambiano 3.928 años

Con respecto a la organización definitiva de la familia, Constenla recomienda lo siguiente: "La propuesta de subagrupación que he hecho (...) deberá compararse con los [resultados] que dé el método comparativo cuando éste se aplique intensamente a la reconstrucción del protobarbacoa. Este método hasta el momento sólo ha sido aplicado (...) a la rama meridional de la familia por Moore (1962)", quien ha propuesto el sistema fonológico del protobarbacoa meridional (LAI: 77).

En el presente trabajo se compararán datos del páez con datos de las lenguas barbacoas awa-cuaiquer y guambiano. La siguiente información sobre estas lenguas aparece en el VC (entre paréntesis, nombres alternativos de la lengua):

AWA (cuaiquer, coaiquer, awa-cuaiquer, kwaiker)

Hablantes: 20.000 .

Residencia: la mayoría en Colombia y algunos en la selvas tropicales de la ladera pacífica de los Andes, en Ecuador.

Inventario fonológico:

Consonantes: $\mathrm{p}, \mathrm{t}, \mathrm{t}\}, \mathrm{k}, \mathrm{2}, \mathrm{s}, \int, \mathrm{x}, \mathrm{h}, \mathrm{z}, \mathrm{m}, \mathrm{n}, \mathrm{n}, \mathrm{l}, \mathrm{w}, \mathrm{j}$.

Vocales: a, e, i, o, u, i (orales y nasales).

GUAMBIANO (guambia, moguez)

Hablantes: 18.000 .

Residencia: Cordillera Central de los Andes cerca de Popayán, Cauca, Colombia. Inventario fonológico:

Consonantes: $\mathrm{p}, \mathrm{t}, \mathrm{k}, \mathrm{k}^{\mathrm{w}}$, ts, tf, tc, $\mathrm{s}, \int, \mathrm{c}, \mathrm{3}, \mathrm{r}, \mathrm{l}, \mathrm{l}^{\mathrm{j}}, \mathrm{m}, \mathrm{n}, \mathrm{n}, \mathrm{w}, \mathrm{j}$.

Vocales: a, e, i, u, i. 


\title{
3.2. La lengua páez
}

Pachón (2000) indica que la población páez se calcula en unas $\mathbf{8 0 . 0 0 0 ~ p e r s o n a s ~ q u e ~}$ residen principalmente en Tierradentro del Departamento del Cauca, Colombia. De acuerdo con LAI, aunque el páez suele asociarse con el paniquitá ${ }^{5}$ y el panzaleo (Loukotka 1968), en ninguno de los dos casos la relación parece justificable. Con respecto a la relación con el guambiano propuesta por Greenberg, Landaburu afirma que "el conocimiento preciso que se está empezando a tener tanto del páez como del guambiano muestra dos lenguas muy diferentes tanto en su léxico como en su estructura" (Ibid.).

Referencias a los materiales descriptivos del páez anteriores a 1970 se hallan en Loukotka (1968) y posteriores en Gerdel $(1973,1983)$ y Gerdel \& Slocum (1976).

El VC presenta la siguiente información sobre la lengua:

\section{PÁEZ (nasa yuwe)}

Hablantes: 68.000 .

Residencia: Cordillera Central de los Andes cerca de Popayán, Cauca.

Inventario fonológico:

Consonantes: $\left.\mathrm{p}, \mathrm{t}, \mathrm{t}^{\mathrm{j}}, \mathrm{k}, \mathrm{q}, \mathrm{b}, \mathrm{d}, \mathrm{d}^{\mathrm{j}}, \mathrm{g}, \mathrm{ts}, \mathrm{t}\right\}, \Phi, \mathrm{s}, \int, \mathrm{x}, \boldsymbol{\beta}, \mathrm{z}, \mathbf{3}, \mathrm{\gamma}, \mathrm{\rho}^{\mathrm{j}}, \mathrm{x}^{\mathrm{j}}, \mathrm{r}, \mathrm{l}, \mathrm{l}^{\mathrm{j}}, \mathrm{m}, \mathrm{n}$, $\mathrm{n}, \mathrm{w}, \mathrm{j}$.

Vocales: a, e, i, u (orales y nasales).

\subsection{La familia chocó}

Pardo (2000) explica cómo se llegó a denominar conjuntamente "chocoes" a los grupos emberá y huaunana en los siguientes términos:

\begin{abstract}
Desde la época de la Conquista se denominó como indígenas Choc a los auto llamados Emberá asentados en los cauces superiores de los ríos Atrato y San Juan. Posteriormente, y dadas las similitudes socioculturales, el apelativo Chocó se amplió para los Waunana del bajo San Juan. Desde finales del siglo XIX el término Chocó ha sido usado para designar tanto al grupo étnico, como al grupo linguístico integrado por los idiomas Emberá y Waunana, que están estrechamente emparentados.
\end{abstract}

De acuerdo con Pardo (Ibid.), la población chocó es aproximadamente de 50.000, de los cuales 42.000 residen en Colombia ( 38.000 emberaes y 4.000 huaunanas) y 8.000 residen en Panamá (6.000 emberaes y 2.000 huaunanas).

Según indica LAI, Nordenskjöld (1929) divide la familia en huaunana (noanamá) (wounaan en el VC) y emberá. Loewen (1960) da un parentesco léxico del $50 \%$ entre ellas, lo que correspondería a una separación de unos 2130 años. Loewen (1963) divide las lenguas emberaes en dos ramas: septentrional (o norte, que incluye los dialectos catío (dabeiba), San Jorge, Río Verde y sambú) y meridional (o sur, que incluye saija, baudó, citará (atrato, Río Sucio), tadó y chamí). El dialecto sambú es de Panamá y los demás son de Colombia.

Algunos indicios sugieren que los cuevas (de la región oriental de Panamá) podrían haber sido chocoes, al menos en parte, y no chibchas, como propuso Lehmann (1920) (LAI: 47). En EFCC también se hace referencia a la posibilidad de que los cuevas de la región oriental de Panamá hayan sido chocoes. "Los cuevas han sido identificados como chibchas, inclu- 
so concretamente, como especialmente afines a los cunas" (EFCC: 138). Para los autores, esta relación no se justifica, especialmente por las observaciones que ha aportado Loewen (1963) con respecto a la posible relación de los cuevas con los chocoes. Uno de los indicios convincentes para los autores es la etimología de 'comer': cueva: chica, emberá: tfkho, cuna: kunne. Puede resultar útil, en caso de una posible relación del páez con las lenguas chocoes, agregar a este conjunto la palabra 'comején' en páez: $\phi t u \tilde{u}$ tfika, de $\phi t u \tilde{u}$ 'madera' y tjika, al parecer una forma de 'comer' relacionada con las formas citadas de cueva y emberá.

De nuevo, la propuesta de Greenberg (1987) de que la familia chocó pertenecería al tronco llamado por él "paezano" (paezan) es para muchos investigadores una agrupación sin fundamento (VC: xvii), y es una de las motivaciones del presente trabajo. La clasificación que sigue el VC es la de Grimes (1988), que incluye seis lenguas en la familia (entre paréntesis la clasificación de la lengua que aparece en Grimes 1988):

1. wounaan (chocó),

2. catío (chocó, embera, norte),

3. embera darién (chocó, embera, norte),

4. embera chamí (chocó, embera, sur),

5. epena saija (chocó, embera, sur) y su dialecto embera basurudó, y

6. embera tado (chocó, embera, sur).

En Grimes (2000), la familia aparece organizada de la siguiente manera:

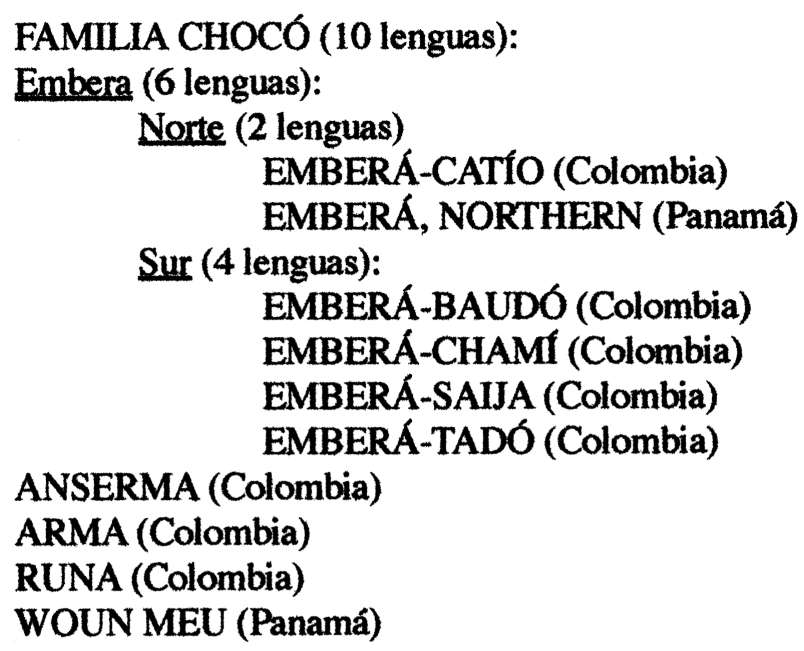

(http://www.ethnologue.com/show_family.asp?subid=1921)

Como ejemplo de lengua viva de esta familia, en este trabajo se tomarán en cuenta datos de la lengua huaunana (nombres alternativos: wounaan, waunana, waunán, noanama, noenama, nonama, chocama, chanco, woun).

El VC presenta el siguiente inventario fonológico del huaunana:

Consonantes: $\mathrm{p}^{\mathrm{h}}, \mathrm{t}^{\mathrm{h}}, \mathrm{k}^{\mathrm{h}}, \mathrm{p}, \mathrm{t}, \mathrm{t} \mathrm{f}, \mathrm{k}, \mathrm{l}, \mathrm{b}, \mathrm{d}, \mathrm{g}, \mathrm{s}, \mathrm{h}, \mathrm{l}, \mathrm{s}, \mathrm{r}, \mathrm{m}, \mathrm{n}, \mathrm{w}, \mathrm{j}$.

Vocales: $a, e, i, o, u, i$ (orales y nasales), además $i, \partial, \Lambda, v$.

En LAI se incluye el inventario fonológico del huaunana según Binder \& Binder 
(1974), el cual coincide con el que se presenta en el VC, excepto por dos diferencias vocálicas que podrían ser dialectales o simplemente de transcripción:

VC:

$a, e, i, o, u, i$ (orales y nasales), $1,2, \Lambda, v$..

Binder \& Binder (en LAI): $\quad$ a, e, i, o, u, u (orales y nasales), $\mathbf{I}, \boldsymbol{\gamma}, \Lambda, v$.

Referencias a los materiales descriptivos de las lenguas chocoes se encuentran en Loewen 1963 (la mayoría de ellos), Loukotka (1968) y Wares (1985).

Con respecto a la diacronía de estas lenguas, LAI apunta la ausencia de estudios diacrónicos para la familia (ver también Constenla y Margery 1991):

Los trabajos realizados con el método de inspección como los de Rivet (1943/4) y Greenberg (1987) o la aplicación de la glotocronología basada en dicho método como en el caso de Swadesh (1959) o Weißhar (1982) se han enfocado en relaciones remotas, proponiendo la inclusión de las lenguas chocoes como rama de la familia caribe (Rivet), el filo macrochibcha (Greenberg) o macroleco (Swadesh), hipotesis de las cuales ninguna todavía puede considerarse probada (LAI: 51 ).

Este trabajo pretende buscar indicios que permitan establecer relaciones entre el páez, la familia chocó y la familia barbacoa.

\title{
4. Posibles cognados entre la lengua páez y las familias barbacoa y chocó
}

\subsection{Motivación}

El presente estudio busca dar un primer paso en respuesta a los siguientes comentarios de Constenla Umaña:

\begin{abstract}
No ha habido hasta el momento aplicación del método comparativo al estudio de las posibles relaciones de parentesco del páez, ninguna de las cuales se ha probado. Las más estrechas se darían, presuntamente, con las lenguas que en este trabajo se han considerado integrantes de la familia barbacoa que, de acuerdo con los estudios glotocronológicos de Swadesh y Weißhar (...), presentarían separaciones de más de 5.000 años con respecto al páez (LAI: 79-80).
\end{abstract}

Es interesante (...) que los porcentajes obtenidos por Wei ßhar para la relación del páez con las lenguas de la familia chibcha (...) promediados den un $10,5 \%$-equivalente (...) a 5.193-, que no difiere gran cosa de los obtenidos tanto por el mismo autor como por Swadesh para la relación del páez con las lenguas barbacoas (LAI: 80).

Por otra parte, el promedio de los resultados de Weißhar en cuanto a la relación del colorado con las lenguas chibchas (...) fue de $14.2 \%$, lo cual plantearía una separación menor (4.497 años) con ellas que con el páez (loc. cit.).

En este trabajo se lleva a cabo la inspección de posibles cognados entre la lengua páez y dos barbacoas, por una parte, y entre el páez y el protochocó, por otra. La inspección se basa en los datos recogidos en el VC para las lenguas páez, guambiano, awa-cuaiquer, huaunana, y algunos del cayapa y el colorado; de EFCC se toman las formas reconstruidas del protochocó y algunas del protoemberá. La comparación que se lleva a cabo es relativa a los 375 términos de las listas comparativas en el VC. 
4.2. Inspección de posibles cognados entre las lenguas barbacoas y el páez

Tabla No. 1-a

\begin{tabular}{|c|c|c|c|}
\hline No. & glosa & GUAMBBIANO & PAEZ \\
\hline$\Gamma$ & ojo & kap & jaф \\
\hline 2 & mentón & kumpampa & kbaba \\
\hline 3 & cuello & nafik & t'hikh \\
\hline 4 & mujer & ifuk & $\mathbf{u} \mathbf{j}$ \\
\hline 5 & pantano & t? jilij $^{j} \mathrm{ik}$ & $\mathrm{t}^{\mathrm{j}} \mathrm{it}^{\mathrm{j}}$ 'barro' \\
\hline 6 & murciélago & $\mathrm{kil}^{\mathrm{j}} \mathrm{itsim}_{\mathrm{its}}$ & khĩtse \\
\hline 7 & culebra & ul & ul \\
\hline 8 & frío & pi $\int \tilde{1}$ & фize \\
\hline 9 & amarillo & iski & Skiikj \\
\hline 10 & calabazo poró & tur & tuka \\
\hline$\Pi$ & cinco & tcattci & tahts \\
\hline & & AWA-CUATQUER & PAEL \\
\hline 12 & teta & tfitfu & t $\int u ? t \int$ \\
\hline 13 & punta (de lengua) & (jankwixia) kwiz & (thune) $\beta$ its \\
\hline 14 & pluma (lit. pájaro pelo) & tfit $\int u$ as & ßitfa-kwe \\
\hline 15 & perico & t $\int i t i t t u$ & $\mathrm{t} \int \mathrm{i}$ ? \\
\hline 16 & Tleno & punta, tuktu & uta \\
\hline 17 & pesado & us & duh \\
\hline 18 & semilla & pippa & фiw \\
\hline 19 & este (demostrativo) & an & naa \\
\hline 20 & donde & minta & m-tee, m-suu \\
\hline 21 & cuatro & ampata & pahz \\
\hline
\end{tabular}

Tomando como fuente los 375 rubros léxicos que se presentan en el VC, se estableció que las parejas de palabras en la tabla No. 1-a podrían estar emparentadas. 
Tabla No. 1-b

\begin{tabular}{|c|c|c|c|c|}
\hline No. & glosa & GUAMBIANO & AWA-CUATQUER & PAEZ \\
\hline 22 & cola & mitc & miti & mez \\
\hline 23 & hueso & tsutsik & tfih & $\mathrm{d}^{\mathrm{j} i \mathrm{i} t}$ \\
\hline 24 & casa & $\overline{j a}$ & jal & jat \\
\hline 25 & piedra & cuk & uk & kwet (1) \\
\hline 26 & pajaro & $\mathrm{il}_{\mathbf{1}}^{\hat{1}}$ & $t \int i t \int u$ & ßitfa-kwe \\
\hline 27 & caliente & patfik & $\mathbf{i}$ & at $\int a$ \\
\hline 28 & seco & pur & pul & uhd $^{\mathrm{j}}$ \\
\hline 29 & botar & wajip & watna & wããtãh- \\
\hline 30 & soplar & ũtçip & usna & puth- (2) \\
\hline 31 & árbol & tuc & $\mathbf{t i}$ & $\oint$ tũ, фtũũtaS \\
\hline 32 & cerro, loma & tun & put tih & thã?h \\
\hline
\end{tabular}

(1) cayapa: skupuka; colorado: so 'piedra'

(2) cayapa: huipunu; colorado: furipo- 'soplar'

En cuanto a los rubros de 1 a 11, no se observaron semejanzas entre las formas del awa-cuaiquer con respecto a las de guambiano y páez, mientras que en los rubros de 12 a 21 tampoco se observaron similitudes entre las formas del guambiano con respecto a las del awacuaiquer y páez, razón por la cual no he incluido dichas formas en la Tabla 1-a. En cambio, los tríos de palabras en la tabla No. 1-b muestran alguna semejanza, por lo que podrían estar emparentados:

Si asumiéramos que en efecto estos 32 términos están emparentados, tendríamos una relación de parentesco léxico entre la lengua páez y la familia barbacoa tomada en su conjunto, de $8.53 \%$; de manera que este sería el máximo grado de parentesco que podría darse entre ambas. Por supuesto, esta es una cifra muy idealizada: no puede obviarse el hecho de que en ninguno de los tres casos comparados (páez-guambiano, páez-awa cuaiquer y páez-guambiano-awa cuaiquer) se llega a alcanzar ni un $3 \%$ de parentesco, y esto solo en el caso de que todos los términos estuvieran emparentados.

\subsection{La familia chocó y la lengua páez}

Otro de los aspectos controversiales de la agrupación propuesta por Greenberg es la inclusión de la familia chocó en el filo denominado por él mismo paezan nuclear (ver 3.1), que incluye, entre otras, a las familias chocó, barbacoa y páez.

En cuanto a formas que muestran alguna semejanza entre la familia chocó y el páez, 
tenemos las siguientes; parece útil consignar las respectivas formas en guambiano y awa-cuaiquer con el fin de que se observen semejanzas y diferencias entre estas y las de la comparación páez-chocó:

Tabla No. 2-a

\begin{tabular}{|c|c|c|c|c|c|}
\hline No. & glosa & $\begin{array}{l}\text { CHOCO } \\
\text { PC: protochocó } \\
\text { PE: protoemberá }\end{array}$ & PÁEZ & GUAMBIANO & AWA \\
\hline$T$ & boca & PC: $*_{i}$ & juwe & tcik & pit \\
\hline 2 & diente & $\mathrm{PC}:{ }^{*} \mathrm{ki}^{\prime} \mathrm{da}$ & ki?h & tcukul & sula \\
\hline 3 & teta & $\mathrm{PC}: * \mathrm{hu}$ & tfu?t & pitsiwac & tfitfu \\
\hline 4 & brazo & PC: *hu'a ('mano') & huse & kwal & sail \\
\hline 5 & corazón & PE: ${ }^{*} \mathrm{SO}$, sõ & ũũs & mantci & kaixsu \\
\hline 6 & humo & PC: *na'ri & ah & nakif & if \\
\hline 7 & grande & PE: *uai'ßua & wala (1) & nu & katsa \\
\hline 8 & pajaro & PC: *it $\int \hat{i}+$ bana & Bitfakwe & $\mathbf{i}^{\mathbf{j}^{\mathbf{j}} \mathbf{i}}$ & $t \int i t \int u$ \\
\hline 9 & pescado & PC: *wa- ('pescar') & wed $^{j}$ & pipilim & pifkatu \\
\hline 10 & frío & PC: *hî'su ('estar frío) & $\phi i z e$ & pifi & tih \\
\hline$\pi$ & nuevo & PC: *iudi & uise & $\mathbf{6 i}$ & masas \\
\hline 12 & delgado & huaunana: nasu & $\mathbf{z u z}$ & $\int i^{j}$ & nas, nasmin \\
\hline 13 & vivo & huaunana: iiu & $\tilde{\mathbf{i}} \mathbf{t}_{\mathbf{i}}^{\mathbf{i}}$ & isik & $\mathbf{k a ̃}$ \\
\hline 14 & beber & PC: *do- & $\operatorname{tud}^{\mathbf{j}}$ & mutfip & kwan \\
\hline 15 & venir & PC: *uru, t $\int e$ & juh & atcup & an \\
\hline 16 & botar & emberá: $\beta a t^{\mathrm{h}} \mathrm{a}-$ & wããtâh- & wajip & watna \\
\hline 17 & soplar & PC: *pua- & puth- & utcip & usna (2) \\
\hline 18 & temer & huaunana: $u g k^{h} a-$ & ũưkh- & kiripkip & iskwan \\
\hline 19 & yuca & emberá: t $\int$ ãsõ & na & lu & $\mathbf{k} \tilde{\mathbf{u}}$ \\
\hline 20 & $\begin{array}{l}\text { este } \\
\text { (demostr.) }\end{array}$ & PE: *nâ'ku & naa & iik & an \\
\hline 21 & que & PC: *'kãre & kilh & $t \int i$ & mika tzi \\
\hline 22 & quien & PC: *'kai & kim & $\mathrm{mu} \min$ & \\
\hline
\end{tabular}

(1) cayapa: aahua; colorado: wa 'grande'

(2) cayapa: huipunu; furipo- 'soplar' 
Si asumiéramos que todos estos términos (más los tres de la Tabla 2-b) están emparentados en efecto -de lo cual expreso mi más firme duda-, la máxima relación léxica que podría proponerse entre las lenguas chocoes en su conjunto y el páez sería de $6.66 \%$, casi $2 \%$ menos que entre el páez y las lenguas barbacoas. Esto pareciera favorecer la opinión de que el páez se relaciona más con la familia barbacoa que con la chocó. Sin embargo, el único indicio que puede plantearse discretamente como una posible correspondencia sistemática entre el páez y las otras lenguas aquí consideradas se dio en la comparación con las formas del protochocó reconstruidas en EFCC:

$$
\text { *protochocó */d/ : páez/j//\# }
$$

Se dan los siguientes tres casos (se indican las formas de huaunana, así como de guambiano, awa-cuaiquer, cayapa y colorado, por lo que pudieran aportar a una interpretación de la presunta correspondencia):

Tabla No. 2-b

\begin{tabular}{|l|l|l|l|l|l|l|l|l|}
\hline No. & glosa & HOAUNANAPROTOCHOCO & PAEZ & GUAMB. & AWA & CAYAPA & COL. \\
\hline 23 & casa & di & *de & jat & ja & jal & ja & ja \\
\hline 24 & ojo & dau & *da'bu & ja $\phi$ & kap & kasu & ka & kaska \\
\hline 25 & agua & du, doh, du & *do & ju? & pi & pi 'río' & pi, pe & pi, pe \\
\hline
\end{tabular}

\section{Conclusión}

En este trabajo se compararon los 375 términos recopilados en el Vocabulario Comparativo (VC, Huber \& Reed 1992) para las lenguas páez, guambiano, awa-cuaiquer, huaunana, y formas del protochocó y el protoemberá reconstruidas en EFCC (Constenla y Margery 1991) con el fin de buscar indicios de la posible relación genética del páez con la familia barbacoa por una parte y con la familia chocó, por otra. Los términos que muestran alguna semejanza y, por ende, podrían estar emparentados fueron 32 en el caso de la comparación páez-barbacoa y 25 en el caso de la comparación páez-chocó. Son cifras muy pequeñas que no permiten plantear con seriedad una relación genealógica en ninguno de los dos casos. La investigación futura deberá buscar más indicios que permitan relacionar las familias chocó y barbacoa, y eventualmente estas con las familias chibcha y arahuaca. Se tratará probablemente de relaciones distantes, de mucho más allá de los 5000 años.

\section{Notas}

1. Información adicional sobre esta obra se encuentra en http://wings.buffalo.edu/linguistics/ssila/books/ indbook/ b433.htm: "All complex forms are provided with an analysis. The compilers have also provi- 
ded a survey of the genetic classification of Colombian languages, phonemic inventories of all languages listed, a full bibliography of sources, indexes, and locator maps. The text is in Spanish and English in parallel columns. The editors state that "although some of the data may be available in other publications, it is our hope that presenting the data in this format will facilitate comparison." (...) Order from: Instituto Lingüístico de Verano, Apartado Aéreo 100602, Bogotá, Colombia."

Información adicional sobre esta obra se encuentra en http://linguistics.buffalo.edu/ssila/books/indbo ok/b177.htm: "An areal-typological study of the languages of Gordon Willey's culturally defined "Intermediate Area," lying between Mesoamerica and the Caribbean, Amazonian, and Andean regions of South America. Extending from eastern Honduras to Ecuador, this territory has the Chibchan language family at its center, but includes also: Lenca, Jicaque, Misumalpan, Choco, Yurumanguí, Timote-cuica, Jirajara, Betoy, Barbacoa, Páez, Camsá, Cofán, Esmeraldas, and portions of Carib, Maipuran (Arawakan), and Quechua. After reviewing the descriptive and historical literature on these languages and language groups, Constenla surveys the data on 26 phonological features and several diagnostic morphosyntactic features (order of elements in the clause; interrogation and negation; pronominal categories; case systems; etc.). He concludes that the "Intermediate Area" falls apart linguistically into three quite distinct sectors: Venezuela-Antilles; Ecuador and Andean Colombia; and Colombia-Central America. This is a well-researched and well-organized book, and C.'s survey of the bibliography on "Intermediate Area" languages is especially useful. - Order from: Editorial de la Universidad de Costa Rica, Ciudad Universitaria Rodrigo Facio, Apdo. 75-2060 San José, Costa Rica."

3. Cayapa

4. Colorado

5. Nótese (sección 3.1) que Landaburu considera el paniquitá un dialecto del páez.

\section{Bibliografía}

Referencias y bibliografía adicional para el estudio de la lengua páez y las familias barbacoa y chocó.

Nota: Las obras que se refieren a lenguas específicas se enlistan según la lengua; el * remite a la sección "Obras generales o colectivas".

Fuentes: Dietrich 2004, Fabre 2004, Grimes 2000, Landaburu 2000.

\section{Obras generales o colectivas}

Baptista, Priscilla M. (ed.). 1974. Sistemas fonológicos. Lenguas de Panamá, 1. Panamá: Instituto Nacional de Cultura / Instituto Linguístico de Verano.

Beuchat, H. \& Paul Rivet. 1910. Affinités des langues du Sud de la Colombie et du Nord de l'Équateur. Louvain: Le Muséon.

Brend, Ruth M. (ed.). 1985. From phonology to discourse: Studies in six Colombian lan guages. Language Data, Amerindian Series, 9. Dallas: Summer Institute of Linguistics.

Brinton, Daniel G. 1891. The American Race. New York: N. D. C. 
Cathcart, Marilyn E. et al. (eds.). 1979. Sistemas fonológicos de idiomas colombianos 4. Bogotá: Ministerio de Gobierno / Instituto Linguiístico de Verano.

Constenla Umaña, Adolfo. 1985. "Clasificación lexicoestadística de las lenguas de la familia chibcha”. Estudios de lingüística chibcha. 4: 155-197. Universidad de Costa Rica.

1991. Las Lenguas del Área Intermedia: Introducción a su Estudio Areal. San José: Editorial de la Universidad de Costa Rica.

Dietrich, Wolf (director del proyecto). 2004. Lingüística Amerindia Sudamericana. Las lenguas indígenas de América. http://www.uni-muenster.de/Romanistik/LAS/welcome.htm\#noroeste.

Elson, Benjamin F. (ed). 1962. Studies in Ecuadorian Indian languages 1. Linguistic series (7). Norman: Summer Institute of Linguistics of the University of Oklahoma.

Fabre, Alain. 2004. Towards a data base for typological studies of South American and Lower Central American indigenous languages (morphology and syntax). http://butler.cc.tut.fi/ fabre/typologydatabase.html

Gerdel, Florence L. et al. 1973. Sistemas fonológicos de idiomas colombianos 2. Bogotá: Ministerio de Gobierno / Instituto Lingüístico de Verano.

González de Pérez, María Stella y María Emilia Rodríguez de Montes (eds). 2000. Lenguas indígenas de Colombia: una visión descriptiva. Bogotá: Instituto Caro y Cuervo.

Greenberg, Joseph H. 1987. Language in the Americas. Stanford: Stanford University Press.

Grimes, Barbara F. (ed.). 1988. Ethnologue: Languages of the World. 11th ed. Dallas: Summer Institute of Linguistics.

(ed.). 2000. Ethnologue: Languages of the World. 14th ed. SIL International. www.ethnologue.com

Gunn, Robert D. (comp.). 1980. Clasificación de los idiomas indígenas de Panamá con un vocabulario comparativo de los mismos. Lenguas de Panamá, 7. Panamá: Instituto Lingüístico de Verano.

Heinze, Carol (ed.) 1978. Estudios chibchas 2. Serie Sintáctica, 9. Bogota: Ministerio de Gobierno / Instituto Lingüístico de Verano.

Huber, Randall Q. \& Robert B. Reed (comps. y eds.). 1992. Vocabulario Comparativo: Palabras Selectas de Lenguas Indígenas de Colombia/Comparative Vocabulary: 
Selected Words in Indigenous Languages of Colombia. Bogotá: Instituto Lingüístico de Verano.

Instituto Colombiano de Antropología. 2000. Introducción a la Colombia Amerindia. Biblioteca Virtual Luis Ángel Arango / Banco de la República. http://www.banrep.gov.co/blaavirtual/letra-a/amerindi.

Jijón y Caamaño, Jacinto. 1940-1947. El Ecuador interandino y occidental antes de la conquista española, 4 Vols. Quito: Editorial Ecuatoriana. Reedición facsimilar: 1998. Quito: Universidad Católica / Abya Yala.

Landaburu, Jon. 2000. Clasificación de las lenguas indígenas de Colombia. Bogotá: Universidad de los Andes. http://www.banrep.gov.co/blaavirtual/letra-1/lengua/clas05.htm

Lehmann, Walter. 1920. Zentral-Amerika, Die Sprachen Zentral-Amerikas. Berlin.

Levinsohn, Stephen H. (ed.). 1978. Estudios sobre el discurso. Lenguas de Panamá, 4. Panamá: Instituto Lingüístico de Verano.

Longacre, Robert E. y Frances M. Woods (eds.). 1976. Discourse grammar: Studies in indigenous languages of Colombia, Panama, and Ecuador, 1. Summer Institute of Linguistics Publications in Linguistics and Related Fields, 52 (1). Dallas: Summer Institute of Linguistics / University of Texas at Arlington.

1977. Discourse grammar: Studies in indigenous languages of Colombia, Panama, and Ecuador, 2. Summer Institute of Linguistics Publications in Linguistics and Related Fields, 52 (2). Dallas: Summer Institute of Linguistics.

Loukotka, Chestmir. 1942. "Klassification der südamerikanischen Sprachen”. Zeitschrift für Ethnologie. 74. Berlín.

1968. Classification of South American Languages. J. Wilbert (ed). Los Angeles: Latin American Center, University of California.

Ortiz, S.E. 1965. "Lenguas y dialectos indígenas de Colombia". En: Historia Extensa de Colombia I. Tomo 3. Bogotá.

Peeke, Catherine M. (ed.). 1975. Estudios fonológicos de lenguas vernáculas del Ecuador. Quito: Ministerio de Educación Pública / Instituto Lingüístico de Verano.

Schauer, Stanley et al. 1973. Aspectos de la cultura material de grupos étnicos de Colombia 1. Bogotá: Ministerio de Gobierno / Instituto Lingüístico de Verano.

Swadesh, Morris. 1959. "Mapas de clasificación lingüística de México y las Américas". Cuadernos del Instituto de Historia, serie Antropológica 8. México. 
Tovar, Antonio. 1961. Catálogo de las lenguas de América del Sur: enumeración, con indicaciones tipológicas, bibliográficas y mapas. Buenos Aires: Editorial Sudamericana.

Tovar, Antonio y Consuelo Larrucea de Tovar. 1984. Catálogo de las lenguas de América del Sur. Gredos: Madrid.

Uhle, Max. 1890. "Verwandtschaften und Wanderungen de Tschibtscha". 7 Congreso Internacional de Americanistas. Berlin.

Wares, A. C. 1985. Bibliography of the Summer Institute of Linguistics. 1985-1986. Dallas: Summer Institute of Linguistics.

\section{Páez (Colombia)}

\section{Nombres alternativos: nasa yuwe}

Gerdel, Florence L. 1973. "Paez phonemes.” Linguistics 104: 28-48. (Versión en español: "Fonemas del páez." En: Gerdel et al 1973*: 7-37.)

1983. "La palabra y la frase fonológica en páez con alusión especial al tono y al acento." Artículos en Lingüística y Campos Afines. 12: 135-55. (Versión en inglés: "Páez: Pitch and stress in the phonological word and phrase." En: Brend (ed) 1985*: 31-42.

Gerdel, Florence L. y Marianna C. Slocum. 1976. "Páez discourse, paragraph and sentence structure.” En: Longacre \& Woods (eds.). 1976*: 259-443. (Versión en español: 1981. "La estructura del discurso, el párrafo y la oración en páez." En: Estudios en páez, v-ix, 1-143. Serie Sintáctica, 12. Bogotá: Ministerio de Gobierno.)

Jung, Ingrid. 1984. Grammatik des Paez. Ein Abriss. Diss. Universität Osnabrück, Alemania. 2000. "El páez. Breve descripción”. En: González de Pérez y Rodríguez de Montes (eds.) 2000*: 139-154.

Pachón, Ximena. 1987. “Páez”. En: Instituto Colombiano de Antropología 2000.* http://www.banrep.org/blaavirtual/letra-a/amerindi/paez.htm

Rojas, Tulio. 2003. “A propósito de los radicales y clases de palabras en la lengua páez”. 51 Congreso Internacional de Americanistas, Santiago de Chile, sección ALL 14.

Slocum, Marianna C. 1972. ¿Cómo se dice en páez? Gramática pedagógica páez-castellano. Lomalinda: Ministerio de Gobierno.

1986. Gramática páez. Lomalinda: Editorial Townsend.

Slocum, Marianna C. \& Florence L. Gerdel. 1983. Diccionario: páez-español / español-páez. Lomalinda: Editorial Townsend. 


\section{Familia barbacoa}

Moore, Bruce R. 1962. "Correspondences in South Barbacoan Chibcha." En: Elson (ed.) 1962*: 270-89.

\section{Awa-cuaiquer (familia barbacoa, Ecuador y Colombia)}

Nombres alternativos: awa, awa pit, cuaiquer, coaiquer, quaiquer, kwaiker.

Calvache Dueñas, Rocío. 2000. "Fonología y aproximación a la morfosintaxis del awa pit". En: González de Pérez y Rodríguez de Montes (eds.) 2000*: 97-116.

Curnow, Timothy Jowan 1997. A grammar of Awa Pit (Cuaiquer): An indigenous language of south-western Colombia. Ph.D. diss., The Australian National University.

2002a. "Conjunct/disjunct marking in Awa Pit". Linguistics. 40: 611-627.

2000b. "Conjunct/disjunct systems in Barbacoan languages". En: Jeanie Castillo (ed.). Santa Barbara Papers in Linguistics, 11. Proceedings of the 4th Workshop on American Indigenous Languages. Santa Barbara: UCSB Department of Linguistics.

Henriksen, Lee A. 1979. "Algunas observaciones sobre un texto cuaiquer." Artículos en Lingüística y Campos Afines. 6: 9-17.

Henriksen, Lee A. y Lynne Henriksen. 1979. "Fonología del cuaiquer." En: Cathcart et al. (eds). 1979*: 49-62.

Henriksen, Lee A. y Pedro V. Obando. 1985. Mane p^nk`h kamshimtus. Una gramática pedagógica del cuaiquer con ejercicios prácticos. Pasto: Universidad de Nariño.

Henriksen, Lee A. \& Stephen H. Levinsohn. 1977. "Progression and prominence in Cuaiquer discourse." En: Longacre \& Woods (eds.). 1977*: 43-67. (Versión en español: 1978. "Progresión y prominencia en el discurso cuaiquer". En: Heinze (ed) 1978*: 49-80.

\section{Guambiano (familia barbacoa, Colombia) \\ Nombres alternativos: guambia, moguez, moguex}

Branks, Thomas H. 1978. "Aspectos relacionados con el fondo y la forma del discurso narrativo alrededor del personaje central, en guambiano". En: Heinze (ed) 1978*: 81-116.

1980. "La cohesión y prominencia en las narrativas del guambiano." Artículos en Lingüística y Campos Afines. 9: 1-91.

Branks, Thomas H. y Judith Branks. 1973. "Fonología del guambiano." En: Gerdel et al 1973*: 39-56. 
1973. “Guambiano.” En: Schauer et al. 1973*: 267-78.

Vásquez de Ruiz, Beatriz. 2000. "Guambiano: algunos aspectos sobre morfología nominal”.

En: González de Pérez y Rodríguez de Montes (eds.) 2000*: 155-168.

\section{Cayapa (familia barbacoa, Ecuador)}

Nombres alternativos: chachi, cha' palaachi.

Abrahamson, Arne. 1962. "Cayapa: Grammatical notes and texts." En: Elson (ed) 1962*: 217-47.

Lindskoog, John \& Ruth M. Brend. 1962. “Cayapa phonemics”. En: Elson (ed.) 1962*: 3144. (Versión en español: "Fonémica cayapa." En: Peeke (ed.) 1975.* 8-20.)

Lindskoog, John y Carrie Lindskoog. 1964. Vocabulario cayapa. Serie de vocabularios indígenas "Mariano Silva y Aceves”, 9. Quito: Instituto Lingüístico de Verano. [Contiene un apéndice gramatical.]

Vittadello, P. Alberto 1988. Cha’palaachi. El idioma cayapa. Miscelánea Antropológica Ecuatoriana, Serie Monográfica 5. Guayaquil: Museo del Banco Central del Ecuador.

Wiebe, Neil. 1977. "The structure of events and participants in Cayapa narrative discourse." En: Longacre y Woods (eds.) 1977*: 191-227.

1985. Añapa’s fables. Quito: Instituto Lingüístico de Verano.

Wiebe, Ruth. 1985. "Demythologization of Cayapa folktales." Notes on Anthropology. 3: 43-48.

\section{Colorado (familia barbacoa, Ecuador) \\ Nombres alternativos: tsafiqui, tsachila}

Dickinson, Connie. 1994. Verbal morphosyntactic structures of Tsafiqui. M.A. Thesis: University of Oregon.

1999. "Semantic and pragmatic dimensions of Tsafiqui evidential and mirative markers”. CLS (35): 29-44. Chicago: Chicago Linguistic Society.

Moore, Bruce 1961. "A statistical morphosyntactic typology study of Colorado (Chibcha)". International Journal of American Linguistics. 27: 298-307. Bloomington, Indiana.

1966. “Diccionario castellano-colorado, colorado-castellano". Llacta. 22: 95-221.

1972. "El sistema fonético del idioma colorado." Boletín de Informaciones Científicas Nacionales. 13: 72-78. Ecuador. 
1979. Método para aprender el idioma colorado, I. Quito: Instituto Lingüístico de Verano.

1991. Patrones gramaticales del colorado (chibcha). Cuadernos Etnolingüísticos, 15. Quito: Instituto Lingüístico de Verano.

\section{Familia chocó}

Constenla, Adolfo y Enrique Margery. 1991. "Elementos de fonología comparada chocó". Revista de Filología y Lingüística de la Universidad de Costa Rica. 17 (1-2): 137-91.

Loewen, Jacob A. 1960. “Dialectología de la Familia Lingüística Chocó”.

1963. “Choco I et Choco II". International Journal of American Linguistics. 29. Indiana University.

Nordenskiöld, Erland. 1929. "Les rapports entre l'art, la religion et la magie chez les indiens Cuna et Chocó". Journal de la Societé des Americanistes, Nouvelle serie. 21: 141158. Paris.

Pardo, Mauricio. 1987. "Regionalización de Indígenas Chocó. Datos Etnohistóricos, Lingüísticos y Asentamientos Actuales". Boletín Museo del Oro. 18: 46-63. Bogotá.

2000. "Indígenas del Chocó”. En: Insituto Colombiano de Antropología. 2000* http://www.banrep.gov.co/blaavirtual/letra-a/amerindi/choco.htm

\section{Huaunana (familia chocó, Colombia y Panamá)}

Nombres alternativos: wuaunana, waumeo, woun meu, waun meo, wounaan, noanama, noenama, nonama, chocama, chanco

Binder, Ronald E. 1977. “Thematic linkage in Waunana discourse”.En: Longacre \& Woods (eds.). 1977.* 159-90.

1978. “Los enlaces temáticos en el discurso waunana”. En Levinsohn (ed.). 1978.*27-68.

Binder, Ronald E. \& Kathleen P. Binder. 1974. "Fonología waunana”. En: Baptista (ed.). 1978.*71-92.

Binder, Ronald, Phillip L. Harms \& Chindío Peña Ismare. 1995. Vocabulario ilustrado. Tomo 2: Wounmeu-Español-Epena Pedee. Bogotá: Asociación Instituto Lingüïstico de Verano.

Sánchez A., Micaela \& Olga Castro G. 1977. Una gramática pedagógica del waunana: primera parte. Lenguas de Panamá, 3. Panamá: Instituto Lingüístico de Verano 
Homer, Nils M. 1963. "Gramática comparada de un dialecto del Chocó (con textos, índice y vocabulario)". Etnologiska Studier. 26: 79-248. Göteborg.

Loewen, Jacob Abraham. 1954. Waunana grammar: a descriptive analysis. M.A. Thesis, Washington University.

Mejía Fonnegra, Gustavo. 2000. "Presentación y descripción fonológica y morfosintáctica del waunana”. En: González de Pérez y Rodríguez de Montes (eds.). 2000*. 85-96.

Emberá (familia chocó, Colombia)

Nombres alternativos: -Comprende dos zonas dialectales: norte (dialectos catío y norteño) y sur (dialectos baudo, chamí, saija y tad6)

Aguirre Licht, Daniel. 1998. Fundamentos morfosintácticos para una gramática emberá. Lenguas Aborígenes de Colombia. Descripciones, 12. Bogotá: CCELA, Universidad de los Andes.

1999a. Emberá. Languages of the World/Materials 2008. Munich/ Newcastle: Lincom Europa.

1999b. Ergatividad, focalización, tematización y topicalización en emberá. Lenguas Aborígenes de Colombia. Memorias, 6: 317-329. Bogotá: CCELA, Universidad de los Andes.

Binder, Ronald, Phillip L. Harms \& Chindío Peña Ismare. 1995. Vocabulario ilustrado. Tomo 2: Wounmeu-Español-Epena Pedee. Bogotá: Asociación Instituto Lingüistico de Verano.

Gralow, Frances L. 1976. “Fonología del chamr”. En: istemas fonologicos de idiomas colom bianos 3, 29-42. Bogotá: Ministerio de Gobierno / Instituto Lingüístico de Verano.

Harms, Judith (comp.). 1993. Vocabulario ilustrado eperã pedeede eperaarã oopata (Vocabulario ilustrado en el idioma epena cultura). Bogotá: Editorial Alberto Lleras Camargo.

Harms, Phillip Lee. 1984. "Fonología del epena pedee (saija)". En: Sistemas fonológicos de idiomas colombianos 5. Lomalinda: Editorial Towsend, 157-201.

1985. “Epena Pedee (Saija): Nasalization”. En: Brend (ed.). 1985*. 13-18.

1994. Epena Pedee Syntax. Studies in the Languages of Colombia, 4. Summer Institute of Linguistics and the University of Texas at Arlington Publications in 
Linguistics, 118. Dallas: Summer Institute of Linguistics / University of Texas at Arlington.

Heinze, Carol (ed.). 1977. Estudios en camsá y catio. Serie Sintáctica, 4. Bogotá: MInisterio de Gobierno.

Hoyos Benítez, Mario Edgar. 2000. "Informe sobre la lengua emberá del río Napipî". En: González de Pérez y Rodríguez de Montes (eds.) 2000.* 73-84.

Morris, Judy. 1978. “The syllable in Eperâ". En: Donald A. Burquest (ed.). Topics in natural generative phonology, 61-69. Research Papers of the Texas SIL at Dallas: Summer Institute of Linguistics.

Mortensen, Charles A. 1999. A Reference Grammar of the Northern Embera Languages. Studies in the Languages of Colombia, 7. Dallas SIL International.

Pinto, Constancio. 1978. "La cultura Katía, su lengua y su cultura". Cultura y Mitología. Tomo 2. Medellín: Editorial Compás.

Rex, Eileen \& Mareike Schöttelndreyer. 1973. "Sistema fonológico del catío". En Gerdel et al 1973.*73-85.

Schötelndreyer, Mareike \& Stephen H. Levinsohn. 1976. "The Catío folktale as a play in acts and scenes". Poetics 5: 247-80. (Versión en español: 1977. "La narración folclórica catía como un drama en actos y escenas"). En: Heinze (ed.). 1977 (ver supra). 95-153.

Schötelndreyer, Mareike. 1979. "Catio”. En: Aspectos de la cultura material de grupos étni cos de Colombia 2: 203-227. Bogotá: Ministerio de Gobierno.

Stansell, David. 1973. “Embera”. En: Schauer et al. 1973.* 179-194. 